\title{
A creche como um local de promoção da saúde à comunidade: um relato de experiência
}

\author{
The daycare as a place of health promotion to the community: an experience report
}

La guardería como lugar de promoción de la salud en la comunidad: un relato de experiencia

Adrianne Carla de Castro Tomé ${ }^{1 *}$, Ana Karla da Silva Dantas ${ }^{1}$, Tamires Castro Chaves ${ }^{1}$, Ana Beatriz Costa da Silva ${ }^{1}$, Sumayla Gabrielle Nascimento da Silva ${ }^{1}$, Renatta Barbosa Marinho ${ }^{1}$, Fábia Helena Teles Miranda ${ }^{1}$, Elusa Maria Paiva Corrêa ${ }^{1}$, Vanessa Yasmin Santos da Costa Alves ${ }^{1}$, Francisco Bruno Teixeira1.

\section{RESUMO}

Objetivo: Relatar e descrever as experiências de discentes de medicina advindas das ações de extensão universitária em creches. Relato de experiência: As intervenções extensionistas foram desenvolvidas por alunos do curso de Medicina em creches tendo como público-alvo crianças da faixa etária de 6 meses a 2 anos, pais e responsáveis e colaboradores das creches, em que se realizou três ações voltadas para os temas sobre higiene bucal e alimentação saudável, com 0 intuito de promoção da qualidade de vida e empoderamento em práticas saudáveis de alimentação e higiene. As atividades educativas realizadas permitiram aos discentes o engajamento no processo educativo de crianças, pais e servidores das instituições, além de contribuir para a estimulação ampla da busca de conhecimento mútuo e promoção da introdução de hábitos saudáveis. Considerações finais: $O$ estudo proporcionou uma aproximação entre a universidade e comunidade, favorecendo um compartilhamento de informações e vivência. Ademais, foi possível verificar a importância de promoção à saúde ao público infantil, visto que fortalece o elo entre família, escola e comunidade.

Palavras-chave: Creches, Educação em saúde, Higiene bucal, Alimentação saudável, Relações comunidade-instituição.

\section{ABSTRACT}

Objective: Report and describe the experiences of medical students arising from university extension actions in day care centers. Experience report: The extension interventions were developed by students of the Medicine course in day care centers targeting children aged 6 months to 2 years, parents and guardians and employees of day care centers, in which three actions were carried out focused on the themes of oral hygiene and healthy eating, with the aim of promoting quality of life and empowerment in healthy eating and hygiene practices. The educational activities carried out allowed students to engage in the educational process of children, parents and servants of the institutions, in addition to contributing to the broad stimulation of the search for mutual knowledge and promoting the introduction of healthy habits. Final considerations: The study provided a rapprochement between the university and the community, favoring the sharing of information and experience. Furthermore, it was possible to verify the importance of health promotion for children, as it strengthens the link between family, school and community.

Key words: Nurseries, Health education, Oral hygiene, Healthy eating, Community-institutional relations.

\footnotetext{
1 Universidade Federal do Pará (UFPA), Altamira - PA.

*E-mail: adriannetome@hotmail.com
} 


\section{RESUMEN}

Objetivo: Informar y describir las experiencias de los estudiantes de medicina derivadas de las acciones de extensión universitaria en las guarderías. Informe de experiencia: Las intervenciones de extensión fueron desarrolladas por estudiantes de la carrera de Medicina en guarderías dirigidas a niños de 6 meses a 2 años, padres y tutores y empleados de guarderías, en las cuales se llevaron a cabo tres acciones enfocadas en los temas de higiene bucal y alimentación saludable, con el objetivo de promover la calidad de vida y el empoderamiento en prácticas de alimentación e higiene saludables. Las actividades educativas realizadas permitieron a los estudiantes involucrarse en el proceso educativo de los niños, padres y servidores de las instituciones, además de contribuir a la estimulación amplia de la búsqueda del conocimiento mutuo y promover la introducción de hábitos saludables. Consideraciones finales: El estudio proporcionó un acercamiento entre la universidad y la comunidad, favoreciendo el intercambio de información y experiencia. Además, se pudo constatar la importancia de la promoción de la salud de los niños, ya que fortalece el vínculo entre la familia, la escuela y la comunidad.

Palabras clave: Viveros, Educación para la salud, Higiene bucal, Alimentación saludable, Relaciones comunidad-institución.

\section{INTRODUÇÃO}

Frente às extensas mudanças envolvendo o mercado de trabalho e suas formas de organização, a mulher, que outrora era absorta em trabalhos domésticos e a criação dos filhos, passou a ter jornadas de trabalho intensas decorrentes da sua inserção no mercado de trabalho. Mediante as necessidades assistenciais das famílias criou-se a creche com a finalidade de auxiliar na guarda e proteção os infantos (FERNANDES FS e DOMINGUES JR, 2017). O Brasil, por sua vez, reflete esse cenário por meio da maior procura das creches e instituições de assistência ao pré-escolar (BARBOSA ALNH e COSTA JSM, 2017).

Acrescidas a essas necessidades, a escola ou creche tem o papel de contribuir para o crescimento da criança no seu período integral, oferecendo condições favoráveis para o aprendizado de forma eficaz, visto que esta é a fase na qual as crianças apresentam maior facilidade na absorção dos conceitos que lhe são ensinados, incluindo as práticas de cuidado à saúde e prevenção de acidentes. Embora o papel da creche seja de grande relevância, também é atribuída à família a função de dar continuidade ao processo educativo iniciado na escola, de forma que possa fortalecer o elo entre escola, família e comunidade (COSTA P, et al., 2019).

Os primeiros anos de vida de um ser humano constituem-se como uma período único de oportunidades para que se tornem membros produtivos da sociedade no futuro, possibilitando assim, o rompimento de ciclos de pobreza e desigualdade. Vista como alguém que está em permanente crescimento e desenvolvimento, a criança representa um grupo prioritário na atenção à saúde da população em consonância com as diretrizes da Agenda de Compromissos para a Saúde Integral da Criança e Redução da Mortalidade Infantil (MACÊDO VC, 2016).

Diante dessa perspectiva, compreende-se que investir em ações promotoras do desenvolvimento deve ser prioridade em uma sociedade que almeja melhorias em sua qualidade de vida. Sendo assim, as creches tornam-se espaços oportunos para a promoção do desenvolvimento infantil e para a busca ativa precoce de agravos da saúde, as quais oferecem valiosas oportunidades para o cuidado promotor do bem-estar físico, social, emocional e cognitivo das crianças nos primeiros anos de vida, fortalecendo a construção da cultura preventiva (MARTINS GS, et al., 2020).

Ademais, ações de extensão em saúde no espaço escolar atuam como um meio eficaz na translocação de saberes e conhecimentos, visto que informações difundidas nessa fase da vida são absorvidas com facilidade, além disso amplia a compressão sobre o cuidado multidisciplinar à criança (COSTA P, et al., 2019). Para isso, utiliza-se como instrumento a Educação em Saúde, o qual tem se configurado como importante para a construção do conhecimento, uma tendência que vem sendo valorizada pela universidade. 
Compreendida como um conjunto de práticas e saberes, a Educação em saúde é voltada à promoção da mudança social dos sujeitos e da comunidade, objetivando a autonomia do usuário na prática do autocuidado, onde proporciona um canal de diálogo com a comunidade e abre um espaço propício para o aporte de subsídios para a melhoria da qualidade de vida da população (VIEIRA MSN, et al., 2021; PAES CCDC e PAIXÃO ANP, 2016; SILVA CB, et al., 2017).

Diante disso, o objetivo do presente estudo consiste em relatar e descrever as experiências advindas das ações de extensão universitária promovidas por um grupo de discentes do curso de graduação em Medicina de uma Universidade da região Xingu no estado do Pará, concretizadas em duas creches.

\section{RELATO DE EXPERIÊNCIA}

Trata-se de um estudo qualitativo, de caráter descritivo, do tipo relato de experiência de um projeto de extensão desenvolvido por discentes de Medicina de uma universidade da região Xingu no estado do Pará. O projeto foi composto de três ações realizadas em duas creches.

As atividades foram elaboradas e desenvolvidas voluntariamente ao longo dos meses de outubro de 2019 e março de 2020 em três etapas: (1) reunião com os coordenadores das creches escolhidas, (2) ação voltada para as crianças e (3) ação voltada para os pais e responsáveis.

Inicialmente, foram realizadas visitas de reconhecimento local, definição do público-alvo, melhor horário para realização das atividades e planejamento multiprofissional para melhor desenvolvimento das ações. Dessa forma, foi possível conhecer as rotinas escolares e identificar as dificuldades e particularidades de cada, possibilitando assim, a execução de estratégias que pudessem sanar as demandas de saúde nas creches.

Os temas a serem abordados foram definidos a partir de diálogos com as educadoras infantis e diretoria da escola, as quais, relataram algumas questões de saúde de maior relevância para o público infantil. Sendo assim, foram definidos dois temas principais: o estímulo à higienização bucal e a promoção à alimentação saudável durante os primeiros anos de vida.

Assim, no período entre outubro de 2019 e março de 2020 foram realizadas duas ações com foco principal no público infantil. A ação realizada na primeira creche teve como tema principal o estímulo à higienização bucal. Como elementos da estratégia metodológica utilizadas para as atividades, destacam-se: brincadeiras lúdicas de teatro com fantoches e demonstração da forma correta de higienização bucal com a escovação em macromodelo odontológico confeccionado pelos próprios participantes do projeto.

Para a segunda ação realizada em outra creche foi abordada a promoção à alimentação saudável durante os primeiros anos de vida. Utilizou-se como estratégia metodológica: teatro e brincadeiras lúdicas onde apresentavam-se os riscos de uma alimentação inadequada.

Durante as atividades extensionistas foi realizada uma ação voltada para os pais e responsáveis, em março de 2020, a qual abordou como temas: a alimentação saudável, a pirâmide alimentar brasileira, os riscos de uma alimentação inadequada durante os primeiros anos de vida, além de demonstrar formas de estimular a criação do hábito de comer frutas e legumes. Para a execução da atividade utilizou-se como elementos metodológicos: palestras explicativas com utilização de slides e rodas de conversas.

\section{DISCUSSÃO}

Inicialmente, foram realizadas reuniões para capacitação dos discentes sobre a finalidade do projeto, público-alvo e atividades que poderiam ser realizadas. Essas reuniões forneceram base para a realização das ações, de forma que, nos encontros foram elencados conceitos sobre a atenção à saúde e o papel da creche como ambiente de discussão à saúde (SILVA MAQ, et al., 2020). Ademais, as atividades realizadas contaram com a participação de crianças, funcionários e responsáveis indicados pela coordenação das creches. 
O ambiente escolar em um contexto de desenvolvimento infantil e de cuidado coletivo gera dúvidas e discussões quanto aos possíveis benefícios e prejuízos advindos do contato entre profissionais da saúde e da educação e outras crianças nos espaços de ensino (BECKER SMS e PICCININI CA, 2019). No entanto, é inegável a relevância da educação infantil em saúde, visto que, fornece conceitos importantes para a formação cidadã saudável através da educação em saúde ao contemplar os princípios do Sistema único de Saúde (SUS) instigando a consciência individual e coletiva em hábitos saudáveis (SILVA MAQ, et al., 2020).

A atividade com as crianças atendidas pela primeira creche consistiu em um teatro de fantoches com personagens que interagiam com o público, cujo objetivo era retratar a importância da escovação dos dentes e das visitas regulares ao dentista, utilizando uma linguagem simples e dinâmica. A ludicidade do teatro se apresenta como um meio eficaz no processo ensino-aprendizagem trazendo como resultado a formação e a melhoria da função motora, intelectual, social ou emocional da criança (BATAGLION GA e MARINHO A, 2019; MINEIRO M e D`ÁVILLA C, 2019).

Durante o teatro, foi esclarecida a necessidade da escovação após cada refeição e antes de dormir, seguindo as recomendações do Ministério da Saúde. Outrossim, foram abordados assuntos referentes aos prejuízos decorrente de uma alimentação rica em doces e industrializados, uma vez que, problemas dentários são mais prevalentes em crianças de baixa renda, influenciados por dieta cariogênica, má higiene bucal e dificuldade do acesso à serviços odontológicos (CARMINATTI M, et al., 2017; NUNES VH e PEROSA GB, 2017).

Ao fim da atividade, foi utilizada uma maquete em formato de boca, confeccionada pelos colaboradores do projeto, para instruir as crianças sobre a escovação correta. Após, houve ampla interação entre as crianças e a maquete visando naturalizar o momento de escovação e desmistificá-lo como um processo doloroso e negativo.

O lúdico constitui um espaço de aprendizado provocando a curiosidade e a imaginação, além de possibilitar a constituição de um campo do prazer funcional, do desafio e da expressão construtiva e relacional. Portanto, o uso de materiais lúdicos, como ferramenta educativa, é primordial para tornar acessível, sobretudo ao público infantil, a elucidação de temas de cunho científico. Além disso, o caráter recreativo por eles proporcionado constituiu um elo comunicativo entre os infantos e os discentes (RIBEIRO KRB, et al., 2020).

É importante destacar que, por meio de observações diretas e de relatos das profissionais das creches, identificou-se que a maioria das crianças não possuem hábitos adequados de higiene, especialmente bucal. A higiene bucal de crianças é acompanhada de muitos estigmas e o medo do dentista é uma condição recorrente na infância. Ademais, pais e responsáveis, a depender da configuração socioeconômica, recorrem a tratamentos odontológicos apenas em casos de emergência, dificultando um controle odontológico preventivo eficaz. Assim, a educação em saúde se torna uma engrenagem essencial, pois com maior conhecimento se dispõem a investir na prevenção de futuros acometimentos em saúde (MUÑOZ V, et al., 2019; PONTE YO, et al., 2020).

O programa de segurança alimentar estabelecido pelo governo federal propõe o acesso de toda a população a uma alimentação adequada e segura, como um direito de todo ser humano (CARDOSO AAR, et al., 2019). Diante dessa perspectiva, a atividade realizada na segunda creche, teve como tema a alimentação saudável e envolveu seis turmas com cerca de 10 alunos cada. Por meio de uma dramatização nomeada como "Teatro das Frutas" incentivou-se o consumo de alimentos saudáveis, enfatizando-se as vitaminas presentes e seus benefícios para o organismo, além de desestimular o consumo de doces e enlatados.

Após a atividade, ocorreu uma dinâmica para avaliar o conhecimento adquirido pelas crianças, a partir do oferecimento de uma cesta com frutas e doces. Pediu-se aos alunos que escolhessem algum alimento e, sempre que escolhiam um alimento saudável, elogiava-se a escolha. O contrário também ocorria, quando uma criança pegava um doce, explicava-se que aquele alimento pode causar problemas, como desconforto abdominal. Observou-se grande participação dos infantos e uma grande curiosidade diante dos alimentos, demonstrando a necessidade e a facilidade de promover a saúde no ambiente escolar. 
Atividade similar foi desenvolvida por Silva AVM, et al. (2016), por meio de ações extensionistas em creches, em Belo Horizonte, onde foram abordados temas como: higiene bucal e corporal, parasitoses e alimentação saudável. O projeto teve como resultados respostas positivas por parte do público-alvo, estimulação ampla da busca de conhecimento mútuo e da promoção à introdução de hábitos saudáveis, fortalecendo assim, a eficácia de projetos voltados a este público.

Atividades extensionistas contribuem para a interligação entre o SUS e a formação em saúde a partir do momento em que ocorre a construção de conceitos e práticas de bem-estar. Nesse sentido, há relevância em conceber uma avaliação da extensão visando identificar fragilidades, projetar ações futuras e identificar mecanismo de aperfeiçoamento e melhorias no planejamento, visto que ações extensionistas geram subsídio para a comunidade e para a formação técnico-científico dos acadêmicos. Verifica-se, portanto, o destaque para a análise dos objetivos definidos na construção do projeto, constatando o alcance das metas (MACEDO DA e BEDRIKOW R, 2019; KALICHMAN AO e AYRES JRCM, 2016).

A atividade destinada aos pais e responsáveis dos alunos e aos educadores contou com a temática "Alimentação infantil nos primeiros anos de vida". A ação iniciou enfatizando-se a importância da amamentação durantes os primeiros meses. Em seguida, explicou-se as recomendações sobre alimentação infantil de acordo com a Sociedade Brasileira de Pediatria. E em seguida, estimulou-se aos pais a aderirem ao lanche da creche, pois, segundo a diretoria, os pais possuem o hábito de enviar lanches industrializados.

Conforme Vasconcelos CMR, et al., (2018), os hábitos de vida dos pais têm efeito direto sobre a rotina das crianças, sobretudo na realização de refeições irregulares, alimentação excessiva e com nutricional deficiente para o desenvolvimento do infanto, além de influenciar no desenvolvimento de um estilo de vida sedentário. Hábitos alimentares inadequados apresentam-se como fator preponderante para o surgimento da obesidade infantil entre outras patologias, sendo este, um empecilho na qualidade de vida dos filhos na infância e também na fase adulta (LINHARES FMM, et al., 2016).

Assim, abordou-se o desenvolvimento de bons hábitos alimentares nas crianças, o uso de habilidades para evitar a rejeição aos alimentos e as consequências a curto e longo prazo da má alimentação infantil, visto que a família é o modelo para o desenvolvimento de preferências e hábitos relacionados à alimentação. Portanto, é importante a instrução familiar quanto a adoção de boas práticas alimentares visando o estímulo adequado a alimentação infantil (DALLAZEN C, et al., 2018). Além disso, deve-se frisar que outros fatores, como a escola, também são ambientes potencialmente modificáveis com poder de influenciar no processo de construção dos hábitos alimentares da criança (LINHARES FMM, et al., 2016).

Segundo a Sociedade Brasileira de Pediatria (2018) a alimentação saudável irá suprir as necessidades nutricionais definidas pelo crescimento pondero-estatural e desenvolvimento neurocognitivo e puberal da criança. Em consonância à isto, por fim, foi ressaltado o risco do surgimento de algumas doenças alimentares como obesidade, bócio, anorexia entre outros, conforme dados do Sistema de Vigilância Alimentar e Nutricional que alertaram sobre o aumento da prevalência de obesidade e sobrepeso em crianças brasileiras entre cinco e dez anos no período entre 2019 à 2020.

Sabe-se que ações extensionistas possuem a capacidade de promover a melhoria na qualidade de vida dos indivíduos envolvidos, auxiliando o conhecimento e empoderando o mesmo. Dessa forma, no que se refere ao público infantil essas atividades possibilitam melhor aprendizagem e impulsionam mudanças no comportamento das crianças (CRUZ JHA, et al., 2019). Assim como, assumindo-se que a falta de informação prejudica fortemente o desenvolvimento da criança, projetos de cunho extensionista impactam positivamente os responsáveis pelo infanto. As creches, por sua vez, beneficiam-se dessas ações por terem como responsabilidade o desenvolvimento de soluções que promovam a associação do cuidar e o educar (COSTA P, et al., 2019).

Por fim, os métodos utilizados nas ações estimularam reflexões e promoveram o autocuidado às crianças das creches, instruindo sobre boas práticas de higiene bucal e escolha adequada de alimentos, prevenindo respectivamente doenças futuras da arcada dentária como cárie e outras infecções e doenças de origem alimentícia como desnutrição, obesidade e diabetes. Além disso, o estudo demonstrou a relevância do 
trabalho realizado pelo projeto extensionista, evidenciando assim, a necessidade de inovação nos métodos utilizados na educação e promoção da saúde. Além de proporcionar uma aproximação entre a universidade e comunidade, favorecendo um compartilhamento de informações e vivências, além de auxiliar para a construção de atividades que sanassem a dificuldade relatada nas creches e contribuindo simultaneamente com a formação ética dos futuros profissionais.

\section{FINANCIAMENTO}

O presente estudo recebeu apoio financeiro da Universidade Federal do Pará, por meio da Pró-Reitoria de Extensão (PROEX), conforme editais PROEX № 05/2019 e PROEX № 06/2019.

\section{REFERÊNCIAS}

1. BARBOSA ALNH, COSTA JSM. Oferta de creche e participação das mulheres no mercado de trabalho no Brasil. Repositório do Conhecimento do IPEA, 2017; 62.

2. BATAGLION GA, MARINHO A. O lúdico em contexto de saúde: inter-relações com as práticas humanizadas. Motrivivência. 2019; 31: 01-19.

3. BECKER SMS e PICCININI CA. Impacto da creche para a interação mãe-criança e para o desenvolvimento infantil. Psicologia: teoria e pesquisa. 2019; 35: e3532.

4. BRASIL. Ministério da Saúde (MS). Política Nacional de Atenção Integral à Saúde da Criança: orientações para implementação. Brasília, 2018. Acessado em 21 de junho de 2021 . Disponível em: https://portaldeboaspraticas.iff.fiocruz.br/wp-content/uploads/2018/07/Pol\%C3\%ADtica-Nacional-de-

Aten\%C3\%A7\%C3\%A3o-Integral-\%C3\%A0-Sa\%C3\%BAde-da-Crian\%C3\%A7a-PNAISC-Vers\%C3\%A3oEletr\%C3\%B4nica.pdf. Acesso em: 21 jun. 2021

5. BRASIL, Ministério da Saúde. Sistema de Vigilância Alimentar e Nutricional. Brasília: MS; 2020. Disponível em: https://sisaps.saude.gov.br/sisvan/relatoriopublico/index. Acesso em: 21 jun. 2021

6. CABRAL RBG, et al. Estudo №2 sobre a evolução do novo Coronavírus (SARS-CoV-2) no estado do Pará (Brasil), Março - Junho, 2020. Brazilian Journal of health Review. 2020; 3: 5397-5426

7. CARDOSO AAR, et al. Educação nutricional para pais e pré-escolares em uma creche. Rev Bras Promoç Saúde. 2019;32:8926.

8. CARMINATTI M, et al. Impacto da cárie dentária, maloclusão e hábitos orais na qualidade de vida relacionada à saúde oral em crianças pré-escolares. Audiol Commun Res, 2017; 22:e1801.

9. COSTA P, et al. Ações de extensão universitária para translação do conhecimento sobre desenvolvimento infantil em creches: relato de experiência. Rev Esc Enferm USP. 2019;53:e03484

10. CRUZ JHA, et al. Atividades de promoção de saúde desenvolvidas por acadêmicos de Odontologia: relato de experiência. ARCHIVES OF HEALTH INVESTIGATION. 2019; 8: 9.

11. DALLAZEN C, et al. Introdução de alimentos não recomendados no primeiro ano de vida e fatores associados em crianças de baixo nível socioeconômico. Cad. Saúde Pública 2018; 34(2):e00202816

12. FERNANDES FS e DOMINGUES JR. Educação infantil no estado de São Paulo: condições de atendimento e perfil das crianças. Educação Pesquisa. 2017; 43 (1): 145-160.

13. KALICHMAN AO, AYRES JRCM. Integralidade e tecnologias de atenção à saúde: uma narrativa sobre contribuições conceituais à construção do princípio da integralidade no SUS. Caderno de Saúde Pública. 2016; 32 : 1-13.

14. LINHARES FMM, et al. Obesidade infantil: influência dos pais sobre a alimentação e estilo de vida dos filhos. Temas em saúde. 2016; 16(2): 460-481.

15. MARTINS GS, et al. Programa saúde na escola: ação educativa promovendo a cultura preventiva no ambiente escolar: relato de experiência. Revista Eletrônica Acervo saúde. 2020; 12(10): 1-6.

16. MACEDO DA, BEDRIKOW R. Projetos de extensão do Curso de Bacharelado em Enfermagem de uma universidade pública brasileira. Saúde em Redes. 2019; 5(3):117-127

17. MACÊDO VC. Atenção integral à saúde da criança: políticas e indicadores de saúde. Recife: Ed. Universitária da UFPE, 2016. 43 p.

18. MINEIRO M, D’ÁVILLA C. Ludicidade: compreensões conceituais de pós-graduandos em educação. Educ. Pesqui, 2019; 45, e208494.

19. MUÑOZ V. Imaginarios, creencias y actitudes que influyen en la consulta odontopediátrica en menores de 3 años. International Journal of Interdisciplinary Dentistry. 2020; 13(1); 06-08. 
20. NUNES VH, PEROSA GB. Cárie dentária em crianças de 5 anos: fatores sociodemográficos, lócus de controle e atitudes parentais. Ciência \& Saúde Coletiva. 2017; 22(1):191-20.

21. PAES CCDC, PAIXÃO ANP. A importância da abordagem da educação em saúde: revisão de literatura. REVASF. 2016; 6:80-90.

22. PONTE YO, et al. Educação em saúde bucal em uma creche pública municipal do interior do Ceará. Revista Eletrônica Acervo Saúde. 2020; 12(3): e2530.

23. RIBEIRO KRB, et al. A influência do lúdico no ensino de enfermagem: uma pesquisa-ação. 2020; $12: 751-757$.

24. SILVA CB, et al. Atividades de educação em saúde junto ao ensino infantil: relato de experiência. Revista de Enfermagem UFPE online. 2017; 11(Supl. 12):5455-63.

25. SILVA AVM, et al. Ações de extensão para promoção da saúde em creche no município de Belo Horizonte, MG. Interagir: pensando a extensão. 2016; (22): 32-45

26. SILVA MAQ, et al. Educação em saúde no contexto da pré-escola: um relato de experiência. Revista Eletrônica Acervo Enfermagem. 2020; 5: 1-5.

27. Sociedade Brasileira de Pediatria. Departamento de Nutrologia Manual de Alimentação: orientações para alimentação do lactente ao adolescente, na escola, na gestante, na prevenção de doenças e segurança alimentar. 4ํa . ed. - São Paulo: SBP, 2018

28. VASCONCELOS CMR, et al. Intervenções educativas na promoção da alimentação saudável em escolares. Rev enferm UFPE online. 2018; 12(10):2803-15.

29. VIEIRA MSN, et al. Educação em saúde na rede municipal de saúde: práticas de nutricionistas. Ciênc. Saúde Colet. $2021 ; 26(02): 12$ 